\title{
Boundary Element Analysis of Reactive Mufflers and Packed Silencers with Catalyst Converters
}

\author{
T. W. Wu \\ Department of Mechanical Engineering, University of Kentucky, Lexington, KY \\ 40506, U.S.A. \\ E-mail: timwu@engr.uky.edu \\ C. Y. R. Cheng \\ Nelson Industries, Inc., Stoughton, WI 53589, U.S.A.
}

\begin{abstract}
This paper reviews recent developments in the application of the boundary element method (BEM) to muffler and silencer analysis. Initial results of modeling built-in catalyst converters are also presented. A so-called "direct mixed-body boundary element method" has been developed for muffler and silencer analysis since 1996. The idea of the method is to integrate all kinds of different boundary and internal surfaces into a single integral equation set without using the conventional multi-domain approach, even though there may be different media in the domain. A key ingredient in this method is the hypersingular integral equation. The concept of the direct mixedbody BEM is not totally against the conventional multi-domain BEM, though. For very large structures or at high frequencies, a multi-domain or substructuring approach is still necessary in order to reduce the memory usage as well as the computation time. With the direct mixed-body BEM, each substructure does not need to be a well-defined and homogeneous subdomain. As such, substructuring can be done more naturally along the longitudinal direction. Catalyst converters may also be modeled as a modular in the general framework of substructuring. Several test cases are presented with experimental verification, including two cases with built-in converters.
\end{abstract}

\section{Introduction}

Reactive mufflers and packed silencers used in industry usually contain very complex internal components such as extended inlet/outlet tubes, thin baffles, perforated tubes, and bulk-reacting sound absorbing materials. Although the interior fluid domain is bounded, the boundary element method (BEM) is still an ideal analysis tool due to its surface-only meshing scheme. Since $1996, \mathrm{Wu}$ and his co-workers $[1,2]$ have used a so-called "direct mixed-body BEM" for the analysis of reactive mufflers with thin internal components and perforated tubes. A key ingredient in the direct mixed-body $\mathrm{BEM}$ is the hypersingular integral equation. The use of the hypersingular integral equation for thin-body scattering problems has been a well-known technique. Back in 1980, Terai [3] presented a regularization technique for 3-D planar (and constant) 
elements to model acoustic scattering from thin plates. Later, Rizzo and his co-workers were among the pioneers to develop general regularization techniques for curvilinear (and higher-order) boundary elements [4-10]. The general regularization procedure for curvilinear elements has enabled more accurate boundary modeling for complex geometry. The regularization technique was adopted in the direct mixed-body BEM to model thin components and perforated tubes inside reactive mufflers [1,2]. It is noted that the modeling of perforated tubes is similar to the modeling of thin bodies except that an empirical transfer impedance is introduced to relate the pressure jump to the normal velocity [11].

For packed silencers, the BEM modeling becomes even more challenging because there are at least two different acoustic media involved, air and the bulk-reacting sound absorbing material. A bulk-reacting sound absorbing material is characterized by a complex speed of sound and a complex mean density [12]. These two material properties are related to the propagation constant and the characteristic impedance which can be measured by the two-cavity method [12] or approximated by empirical formulas if the flow resistivity of the material is known [13]. The traditional modeling approach is to use the multi-domain BEM [14] in which each subdomain contains only one material. However, the internal geometry of a typical packed silencer is usually quite complex and there may be too many subdomains intricately connected together. It is impractical to define so many subdomains and try to match the boundary conditions at all interfaces. To overcome the modeling difficulty, $\mathrm{Wu}$ and his coworkers $[15,16]$ have recently extended the direct mixed-body BEM to packed silencers. The idea is similar to the technique used for reactive mufflers. Boundary integral equations for different subdomains (including those with different material properties) are summed up first. Hypersingular integral equations are then added at interfaces to make up for any missing equations. Because the integral equations for all subdomains are summed up, a multi-domain problem can now be solved in a singledomain fashion. There is no need to define different subdomains or artificial interfaces anymore.

Figure 1 shows a typical packed silencer configuration that can be solved by the direct mixed-body BEM. With reference to the figure, $\Omega$ denotes the interior acoustic domain of the silencer. The symbols R, T, P, B, I, IP, IPC, ATB, and BTB represent "Regular", "Thin", "Perforated", "Bulk-reacting", "Interface", "Interface with Perforated surface", "Interface with Perforated surface and Cloth", "Air-Thin-Bulk-reacting", and "Bulkreacting-Thin-Bulk-reacting" surfaces, respectively. The $\mathrm{R}$ surfaces include the exterior silencer surfaces (with no bulk-reacting packing), the external inlet/outlet tubes, and the inlet/outlet ends. The $\mathrm{T}$ surfaces are the thin components inside the silencer, such as the extended inlet/outlet tubes, thin baffles, thin flow plugs, and internal connecting tubes. Note that a $\mathrm{T}$ surface has air on both sides of the thin surface. The $\mathrm{P}$ surfaces are designated for perforated tubes or any perforated thin plates with air on both sides. The B surfaces are the exterior boundary surfaces of the bulk-reacting lining. The I surfaces are the interfaces between the bulk-reacting material and air. The IP surfaces are the perforated interfaces between the bulkreacting material and air. The IPC interfaces are the same as the IP interfaces except 
that a layer of protective cloth is inserted between the perforated plate and the bulkreacting material to protect the bulk-reacting material from being blown out [16]. The ATB surfaces represent any rigid thin plates between the bulk-reacting material and air. The BTB surfaces represent any embedded rigid thin plates with the bulk-reacting material on both sides.

One unique feature of all direct boundary integral formulations is that it is permissible to use discontinuous elements. With discontinuous elements, a BEM model can be easily assembled from components without worrying about junction continuity, and the mesh can be automatically refined at each frequency. Curvilinear constant elements have been used in the direct mixed-body BEM for muffler and silencer analysis. A coarse mesh is usually used for low-frequency analysis, and the mesh is gradually refined by a simple algorithm as the frequency goes up. This can save a tremendous amount of CPU time as well as mesh preparation time. It should be noted that the surface type naming (such as R, T, P, B, etc in Figure 1) in the direct mixed-body BEM is easy to do and does not require extra preprocessing efforts. When a piece of component mesh is being assembled to the model, its surface type can be attached at the same time. As the mesh is automatically refined later at higher frequencies, the surface type will be carried over.

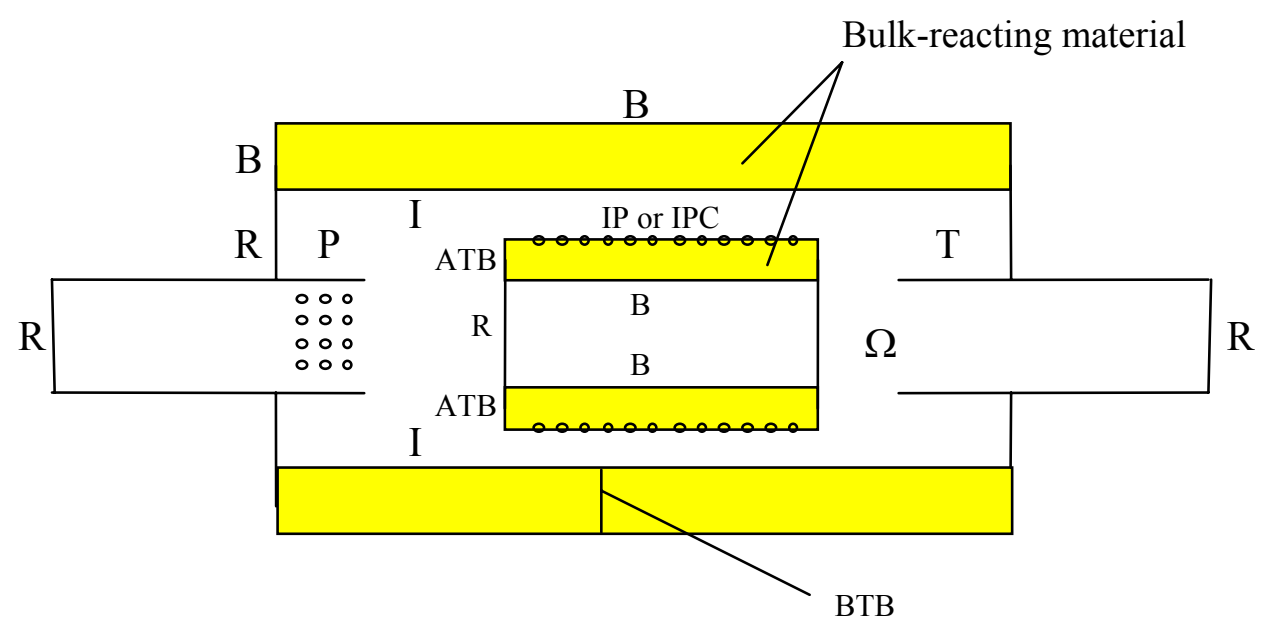

No fluid outside the silencer

Figure 1 Types of surfaces modeled in the direct mixed-body BEM

It should be noted that when the direct mixed-body BEM for muffler analysis was first presented in 1996 [1], it was meant to eliminate the tedious multi-domain BEM zoning and interface matching so that a muffler model with complicated internal components could be created and solved quickly in a single computation. However, as frequency and the number of elements increase, a large silencer model cannot be computed on a PC or workstation anymore mainly due to the physical limitation of available computer memory, and also partially due to the incredibly long CPU time it will take to finish the 
computation. Therefore, dividing a large silencer into smaller substructures so that each substructure can fit into the computer memory one at a time becomes necessary. The BEM first computes the impedance matrix of each substructure one by one. Then, the impedance matrices for substructures are combined by a synthesis procedure to form the resultant impedance matrix for the whole structure [17]. It is noted that in reality the substructuring procedure is nothing but the multi-domain BEM implemented in a modular way. This may sound like some kind of reincarnation of the old multidomain BEM which we have tried very hard to get rid of and replace with the direct mixed-body BEM. Fortunately, the concept of the direct mixed-body BEM is not totally against the multi-domain BEM, though. In a very recent implementation [18], it is shown that the direct mixed-body BEM actually facilitates the substructuring operations. With the direct mixed-body BEM, each substructure may contain very complex internal components (such as thin and perforated components) as well as bulkreacting materials. As a result, substructuring can be done more naturally in the silencer's longitudinal direction. The traditional multi-domain BEM rule of having to construct homogeneous (single-medium) and well defined (no thin or perforated surfaces) subdomains are no longer required. That means substructuring can be done more naturally and elegantly with the help of the direct mixed-body BEM.

The substructuring technique introduced in Ref. [18] may also be applied to mufflers and silencers with an inserted acoustic filter element such as a catalytic converter (CC) or a diesel particulate filters (DPF) [19]. A CC or PDF consists of a stack of capillary tubes inserted between two connecting ducts (substructures). Monoliths used in a CC are categorized as through-flow monoliths in which the substrate is made up by a large number of small parallel tubes (or cells) allowing exhaust gas to flow from one end to the other. The substrate is coated with a layer of wash coat material and catalyst to enhance the catalytic function. As the exhaust gas flows through the catalyst, gaseous pollutants such as nitrous oxide (NOx), carbon monoxide (CO), hydrocarbons (HC) are converted into less harmful gases. Monoliths used in DPF are known as wall-flow monoliths in which exhaust gas is forced to flow through porous tube walls. The removal of soot particles is accomplished by the filtration process in which the wall pores filter out the particulate. Figure 2 shows the comparison between a $\mathrm{CC}$ and a DPF.

To model a CC, a four-pole transfer matrix can be used to represent the connection between the two connecting substructures (one before the $\mathrm{CC}$ and one after). As will be shown in this paper, the transfer matrix is a diagonal matrix with an appropriate set of four-pole parameters derived from the one-dimensional plane-wave theory. The characteristic impedance and the propagation constant of the converter can be measured by the two-cavity method. The diagonal matrix actually represents an "element-to-element" connection between the two connecting substructures separated by the CC. Compared to the CC, the DPF is harder to model due to its wall-flow nature. Rigorously speaking, the one-dimensional plane-wave theory is actually not valid inside the DPF because of the alternate plugs at the inlet and outlet ends. Nevertheless, if we assume that sound waves will penetrate only the neighboring capillary tubes and quickly get out from there due to the least resistance, then the 1-D 
"element-to-element" four-pole connection may still be a good approximation for the DPF.

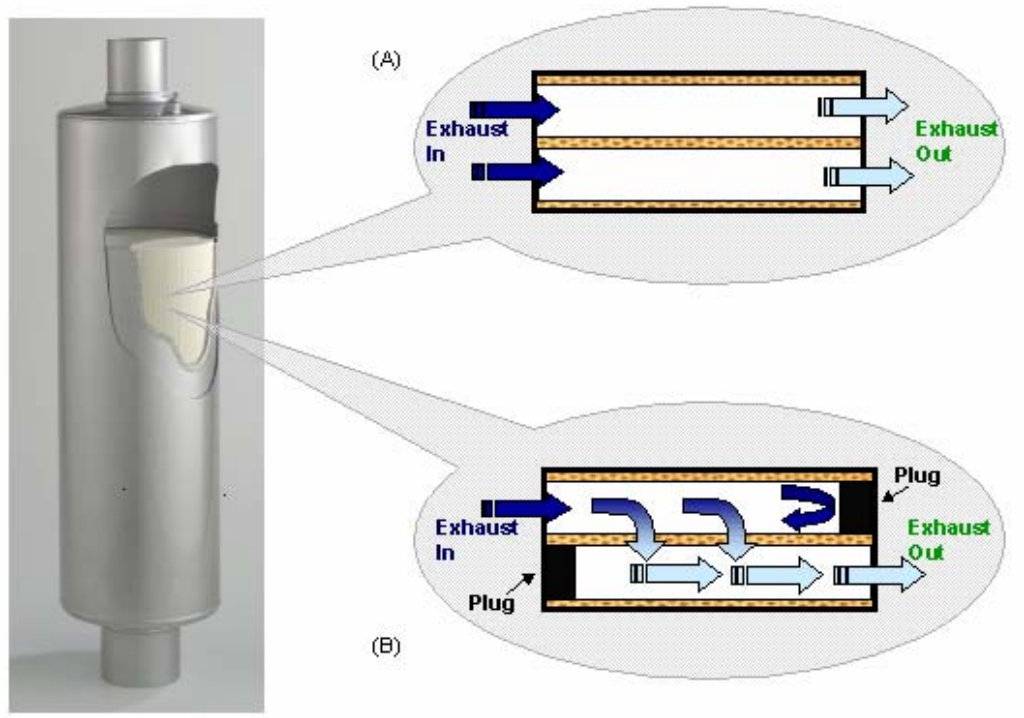

Figure 2 Emission control device with (A) a through-flow catalyst converter (CC), (B) a wall-flow diesel particulate filter (DPF).

In this paper, we first present the boundary integral equations for the direct mixed-body BEM followed by the impedance matrix synthesis used in the substructuring BEM. The connection between two neighboring substructures can be either a four-pole transfer matrix (for a CC or DPF) or simply an identity matrix (for direct connection). Several test cases including two with an inserted CC/DPF are presented to demonstrate the substructuring technique. Numerical results are compared to experimental measurements.

\section{Direct Mixed-Body BEM}

The governing differential equations for a two-medium acoustic problem as depicted in Figure 1 are

$$
\begin{array}{ll}
\nabla^{2} p+k_{A}^{2} p=0 & \text { in air } \\
\nabla^{2} p+k_{B}^{2} p=0 & \text { in the bulk-reacting material }
\end{array}
$$

where $p$ is the sound pressure, and $k_{A}$ and $k_{B}$ are the wavenumbers in air and the bulk-reacting material, respectively. The corresponding fundamental solutions are 


$$
\begin{aligned}
& \psi_{A}=\frac{e^{-i k_{A} r}}{r} \\
& \psi_{B}=\frac{e^{-i k_{B} r}}{r}
\end{aligned}
$$

where $i=\sqrt{-1}$ and $r$ is the distance between the collocation point $P$ and any surface point $Q$.

Let $\omega$ be the angular frequency, $\rho_{A}, \rho_{B}, c_{A}$, and $c_{B}$ be the mean density and speed of sound of air and the bulk-reacting material, respectively. Let $\boldsymbol{n}$ be the unit normal vector. The normal vector on the $\mathrm{R}$ or B boundary is pointing into the interior acoustic domain, and on the I, IP, IPC, and ATB interfaces the normal vector is pointing into the air side. On T, P, and BTB, the normal vector can be pointing into either side; the side into which the normal is pointing is called the positive side, and the corresponding pressure is denoted by $p^{+}$. The other side of T, P. or BTB is called the negative side and the pressure there is denoted by $p^{-}$. Without going through the derivation details, the complete boundary integral equations are given in the following $[15,16,18]$ :

$$
\begin{aligned}
& \int_{\mathrm{R}}\left(p \frac{\partial \psi_{A}}{\partial n}+i \rho_{A} \omega v_{n} \psi_{A}\right) d S+\int_{\mathrm{T}+\mathrm{P}} \frac{\partial \psi_{A}}{\partial n}\left(p^{+}-p^{-}\right) d S \\
& +\int_{\mathrm{B}}\left(p \frac{\partial \psi_{B}}{\partial n}+i \rho_{B} \omega v_{n} \psi_{B}\right) d S+\int_{\mathrm{BTB}} \frac{\partial \psi_{B}}{\partial n}\left(p^{+}-p^{-}\right) d S \\
& +\int_{\mathrm{I}}\left[p\left(\frac{\partial \psi_{A}}{\partial n}-\frac{\partial \psi_{B}}{\partial n}\right)+i \omega v_{n}\left(\rho_{A} \psi_{A}-\rho_{B} \psi_{B}\right)\right] d S \\
& +\int_{\mathrm{IP}+\mathrm{IPC}}\left[p_{A}\left(\frac{\partial \psi_{A}}{\partial n}-\frac{\partial \psi_{B}}{\partial n}\right)-\rho_{A} c_{A} \xi^{*} v_{n} \frac{\partial \psi_{B}}{\partial n}+i \omega v_{n}\left(\rho_{A} \psi_{A}-\rho_{B} \psi_{B}\right)\right] d S \\
& +\int_{\mathrm{ATB}}\left(p_{A} \frac{\partial \psi_{A}}{\partial n}-p_{B} \frac{\partial \psi_{B}}{\partial n}\right) d S
\end{aligned}
$$

$$
=\left\{\begin{array}{cc}
4 \pi p(P) & P \in \Omega \\
2 \pi p(P) & P \in \mathrm{R}+\mathrm{B} \\
2 \pi\left[p^{+}(P)+p^{-}(P)\right] & P \in \mathrm{T}+\mathrm{P}+\mathrm{BTB} \\
4 \pi p(P) & P \in \mathrm{I} \\
4 \pi p_{A}(P)+2 \pi \rho_{A} c_{A} \xi^{*} v_{n}(P) & P \in \mathrm{IP}+\mathrm{IPC} \\
2 \pi\left[p_{A}(P)+p_{B}(P)\right] & P \in \mathrm{ATB}
\end{array}\right.
$$


and

$$
\begin{aligned}
& \int_{\mathrm{R}}\left(p \frac{\partial^{2} \psi_{A}}{\partial n \partial n^{P}}+i \rho_{A} \omega v_{n} \frac{\partial \psi_{A}}{\partial n^{P}}\right) d S+\int_{\mathrm{T}+\mathrm{P}} \frac{\partial^{2} \psi_{A}}{\partial n \partial n^{P}}\left(p^{+}-p^{-}\right) d S \\
& +\int_{\mathrm{B}}\left(p \frac{\partial^{2} \psi_{B}}{\partial n \partial n^{P}}+i \rho_{B} \omega v_{n} \frac{\partial \psi_{B}}{\partial n^{P}}\right) d S+\int_{\mathrm{BTB}} \frac{\partial^{2} \psi_{B}}{\partial n \partial n^{P}}\left(p^{+}-p^{-}\right) d S \\
& +\int_{\mathrm{I}}\left[p\left(\frac{\partial^{2} \psi_{A}}{\partial n \partial n^{P}}-\frac{\partial^{2} \psi_{B}}{\partial n \partial n^{P}}\right)+i \omega v_{n}\left(\rho_{A} \frac{\partial \psi_{A}}{\partial n^{P}}-\rho_{B} \frac{\partial \psi_{B}}{\partial n^{P}}\right)\right] d S \\
& +\int_{\mathrm{IP}+\mathrm{IPC}}\left[p_{A}\left(\frac{\partial^{2} \psi_{A}}{\partial n \partial n^{P}}-\frac{\partial^{2} \psi_{B}}{\partial n \partial n^{P}}\right)-\rho_{A} c_{A} \xi^{*} v_{n} \frac{\partial^{2} \psi_{B}}{\partial n \partial n^{P}}+i \omega v_{n}\left(\rho_{A} \frac{\partial \psi_{A}}{\partial n^{P}}-\rho_{B} \frac{\partial \psi_{B}}{\partial n^{P}}\right)\right] d S \\
& +\int_{\text {АТВ }}\left(p_{A} \frac{\partial^{2} \psi_{A}}{\partial n \partial n^{P}}-p_{B} \frac{\partial^{2} \psi_{B}}{\partial n \partial n^{P}}\right) d S \\
& =\left\{\begin{array}{cc}
0 & P \in \mathrm{T}+\mathrm{BTB}+\mathrm{ATB} \\
4 \pi \frac{i k_{A}}{\xi}\left[p^{+}(P)-p^{-}(P)\right] & P \in \mathrm{P} \\
-2 \pi i \omega\left(\rho_{A}+\rho_{B}\right) v_{n}(P) & P \in \mathrm{I}+\mathrm{IP}+\mathrm{IPC}
\end{array}\right.
\end{aligned}
$$

where $\xi$ is the dimensionless transfer impedance for perforated tubes or perforated surfaces [11], and $\xi^{*}$ is the dimensionless transfer impedance for IP or IPC interfaces. On an IP interface, $\xi^{*}$ is identical to $\xi$. On an IPC interface, $\xi^{*}$ is the sum of $\xi$ and the dimensionless transfer impedance of the protective cloth [16].

\section{Substructuring Using the Impedance Matrix Synthesis (IMS)}

To begin with, we assume that a silencer is divided into two substructures as shown in Fig. 3. It is possible that the two substructures are connected to each other by a builtin acoustic filter element represented by the dotted lines in Fig. 3. Let $p_{i}$ and $v_{i}$ denote the sound pressure and the particle velocity in the longitudinal direction, respectively, at the inlet of the first substructure, and $p_{o}$ and $v_{o}$ denote the corresponding variables at the outlet of the second substructure. Also, $p_{l}$ and $v_{l}$ are the variables at the outlet of the first substructure, and $p_{2}$ and $v_{2}$ are at the inlet of the second substructure. It should be noted that sound pressure and particle velocity may not be uniform at any cross section. Therefore, each $p$ and $v$ variable used in this section actually represents a vector. The length of each vector depends on the number of elements used at each cross section. 
For substructure 1, the sound pressures at the inlet and the outlet are related to the corresponding particle velocities by an impedance matrix

$$
\left[\begin{array}{l}
p_{i} \\
p_{1}
\end{array}\right]=\left[\begin{array}{ll}
Z_{11} & Z_{12} \\
Z_{21} & Z_{22}
\end{array}\right]\left[\begin{array}{l}
v_{i} \\
v_{1}
\end{array}\right]
$$

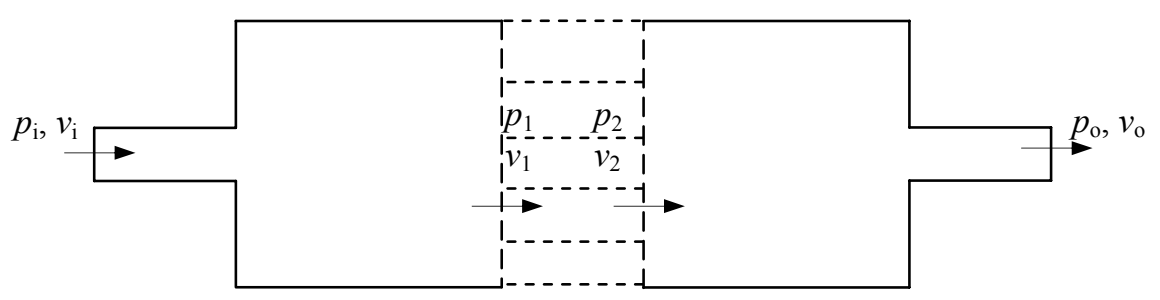

Substructure $1 \quad$ Acoustic filter element $\quad$ Substructure 2

Figure 3 Two substructures connected by an acoustic filter element.

It is important to note that the linear relationship of the above equation is true only when the rest of the boundary of substructure 1 is either rigid or just has an impedance boundary condition. To obtain the impedance matrix, one will need to run the BEM on substructure 1 with a multiple of velocity boundary conditions. For example, to obtain the first column of the impedance matrix, $v=1$ is applied to the first element at the inlet of substructure 1 , and $v=0$ is applied elsewhere. The sound pressure solutions at the inlet and outlet will become the first column of the impedance matrix. Similarly, by making $v=1$ on each element at the inlet and outlet one at a time, the whole impedance can be obtained. It seems that to obtain the complete impedance matrix of Eq. (7) one would have to run the BEM on the substructure for a multiple of times, with a different velocity boundary condition each time. However, it should be noted that only one BEM matrix needs to be formed and decomposed at each frequency. The vectors on the right-hand side of the matrix corresponding to different velocity boundary conditions can be generated at the same time, and each different right-hand side vector only requires a trivial back substitution.

Similarly, one can create the impedance matrix for substructure 2. The impedance matrix relationship is

$$
\left[\begin{array}{l}
p_{2} \\
p_{o}
\end{array}\right]=\left[\begin{array}{ll}
Z_{31} & Z_{32} \\
Z_{41} & Z_{42}
\end{array}\right]\left[\begin{array}{l}
v_{2} \\
v_{o}
\end{array}\right]
$$

For the acoustic filter element that connects the two substructures, a four-pole transfer matrix is used to describe the acoustic property of the filter element. That is, 


$$
\left[\begin{array}{l}
p_{1} \\
v_{1}
\end{array}\right]=\left[\begin{array}{ll}
A & B \\
C & D
\end{array}\right]\left[\begin{array}{l}
p_{2} \\
v_{2}
\end{array}\right]
$$

Note that for a through-flow type catalytic monolith, each capillary tube can be assumed to have a rigid wall. Since the diameter of each capillary tube is small, a plane-wave motion that consists of a forward traveling wave and a backward reflective wave is sufficient to describe the behavior in each capillary tube. Due to the rigid-wall assumption, there is no interaction between any two tubes. Under such circumstances, the transfer matrix coefficients $A, B, C$, and $D$ are each a diagonal matrix. In other words,

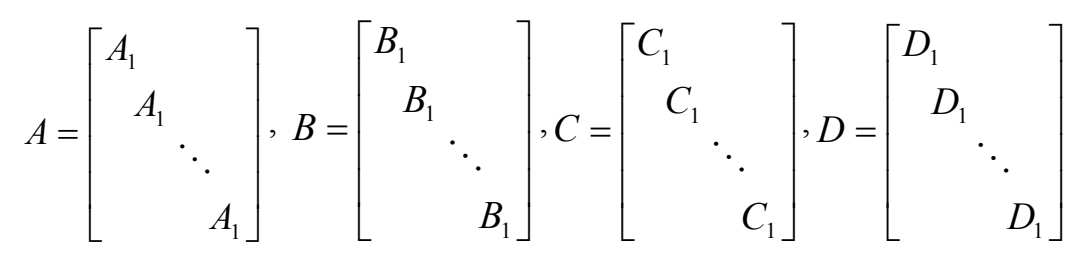

where $A_{1}, B_{1}, C_{1}$, and $D_{1}$ are the four-pole parameters of a straight tube. With the diagonal matrices in Eq. (10), Eq. (9) actually represents an "element-to-element" connection between the two substructures. To account for resistance and friction loss, the four-pole parameters are expressed in terms of a complex mean density $\rho^{*}$ and a complex speed of sound $c^{*}$. Both material properties can be measured by the twocavity method on a sample of CC or DPF with the same length (as in real applications). The four-pole parameters for a straight tube are

$$
\begin{aligned}
& A_{1}=\cos k^{*} L \\
& B_{1}=i \rho^{*} c^{*} \sin k^{*} L \\
& C_{1}=\frac{i}{\rho^{*} c^{*}} \sin k^{*} L \\
& D_{1}=\cos k^{*} L
\end{aligned}
$$

where the complex wavenumber $k^{*}=\omega / c^{*}$ and $L$ is the length of the CC or DPF.

It should be noted that when the two substructures are in direct contact (without the acoustic filter element in between), the transfer matrix reduces to the identity matrix. In other words, $A=D=I$ and $B=C=0$, where $I$ is the identity matrix.

The interface variables, $p_{1}, v_{1}, p_{2}$, and $v_{2}$, can be eliminated from Eqs. (7)-(9) so that $p_{\text {i }}$ and $v_{\mathrm{i}}$ are directly related to $p_{\mathrm{o}}$ and $v_{\mathrm{o}}$. The result is 


$$
\left[\begin{array}{l}
p_{i} \\
p_{o}
\end{array}\right]=\left[\begin{array}{ll}
Z_{51} & Z_{52} \\
Z_{61} & Z_{62}
\end{array}\right]\left[\begin{array}{c}
v_{i} \\
v_{o}
\end{array}\right],
$$

where

$$
\begin{aligned}
& Z_{51}=Z_{11}+Z_{12}\left[C\left(Z_{31} K^{-1} Z_{21}\right)+D K^{-1} Z_{21}\right] \\
& Z_{52}=Z_{12} C\left[Z_{32}-Z_{31} K^{-1}\left(A-Z_{22} C\right) Z_{32}\right]-Z_{12} D\left[K^{-1}\left(A-Z_{22} C\right) Z_{32}\right] \\
& Z_{61}=Z_{41} K^{-1} Z_{21} \\
& Z_{62}=Z_{42}-Z_{41} K^{-1}\left(A-Z_{22} C\right) Z_{32}
\end{aligned}
$$

with

$$
K=\left[\left(A-Z_{22} C\right) Z_{31}+\left(B-Z_{22} D\right)\right]
$$

Note that sound pressure and particle velocity are usually uniform across any cross section in the inlet tube and in the outlet tube as well. That means each of the vectors $p_{i}, v_{i}, p_{o}$, and $v_{o}$ can be lumped into one single variable, and the impedance matrix in Eq. (12) can be further reduced to a $4 \times 4$ impedance matrix. With the lumped $4 \times 4$ impedance matrix ready, the TL of the silencer can be easily obtained [2].

As mentioned earlier, when the two substructures are in direct contact, $A=D=I$ and $B=C=0$. Equation (14) reduces to

$$
K=Z_{31}-Z_{22}
$$

and Eqs. (13a-d) become

$$
\begin{aligned}
& Z_{51}=Z_{11}+Z_{12}\left(Z_{31}-Z_{22}\right)^{-1} Z_{21} \\
& Z_{52}=-Z_{12}\left(Z_{31}-Z_{22}\right)^{-1} Z_{32} \\
& Z_{61}=Z_{41}\left(Z_{31}-Z_{22}\right)^{-1} Z_{21} \\
& Z_{62}=Z_{42}-Z_{41}\left(Z_{31}-Z_{22}\right)^{-1} Z_{32}
\end{aligned}
$$

If a silencer is divided into multiple substructures (more than two) in series connection, the impedance matrix synthesis can be first applied to the first two substructures to produce a resulting impedance matrix. Then the resulting impedance matrix is combined with the impedance matrix of the third substructure by the same synthesis method. The procedure is repeated until the last substructure is reached. It is noticed that many packed silencers in practical use may have an identical cross section in a large portion of the silencer structure. For those kinds of silencers, the impedance matrix of a small section of the identical geometry can be used as a "template", which can be repeatedly used in the synthesis procedure.

\section{Test Cases}


The first test case is a parallel-baffle silencer [18] as shown in Fig. 4. Both the inlet and the outlet tubes have a diameter of 5". The two transition ducts that connect the inlet/outlet tubes to the parallel-baffle section have a square cross section of varying dimensions.

Figure $4 \mathrm{~b}$ shows the cross section of the parallel-baffle section. Two 4"x24"x36" Polyester parallel baffles are used as center splitters in a 24"x24"x36" rectangular duct, and two 2"x24"x36" baffles are attached to the two side walls, respectively, as linings. This creates three 4" air gaps in the center. As shown in Fig. 4c, each Polyester splitter is covered by a perforated metal sheet (designated as IP surface) on its sides and a rigid plate (designated as ATB surface) at both ends. The porosity of the IP interfaces is $23 \%$.

Since the model has two planes of symmetry, only a quarter of the silencer geometry has to be modeled. Even so, the whole model still cannot fit in a PC with 1GB memory at high frequencies without dividing the silencer into smaller substructures. It is noticed the parallel-baffle section has an identical cross section over its length except at the two ATB ends (covering plates). Thus, a template as shown in Fig. 5 can be created and its impedance matrix, once computed for each frequency, can be repeatedly used in the synthesis procedure. Figure 6 shows the comparison of the BEM predictions using four and ten substructures and the experimental TL curve. It is seen that the BEM results compare fairly well with the measured TL curve, although some small discrepancies do exist between models with different number of substructures.

The second test case is a catalytic converter (CC) consisting of a ceramic monolith as shown in Fig. 7 [19]. The catalytic monolith is made up of capillaries with square cross-sections and has a cell density of 400 cells per square inch (CPSI). The monolith used in this study is uncoated. A circular substrate sample is cored out from the catalytic monolith in full length for measuring its characteristic impedance and propagation constant using the two-cavity method. There are two ways to make BEM predictions. One is to directly model the $\mathrm{CC}$ as a block of homogeneous and isotropic bulk-reacting material (BRM), and the other is to model the CC by the "element-toelement" four-pole transfer matrix as described in the impedance matrix synthesis (IMS) section of this paper. As shown in Fig. 8, both BEM predictions compare reasonably well with the experimental data. Both numerical approaches produce very similar results. The IMS approach, however, generates numerical results much faster than the direct BRM modeling method. Since it is assumed that the acoustic propagation in the catalytic monolith is predominantly one-dimensional, a simple fourpole transfer matrix would be adequate to represent the catalytic monolith. The predicted TL curve for an empty housing without the catalytic monolith is also plotted in the same graph for comparison. The results reveal that the first two domes of the TL curves are the reactive effect caused by wave reflections due to area changes in the housing. Beyond the first two domes, the noise attenuation is dominated by the dissipative effect inside the capillaries of the monolith. The catalytic monolith increases the overall TL by more than $5 \mathrm{~dB}$ except for the first dome. 


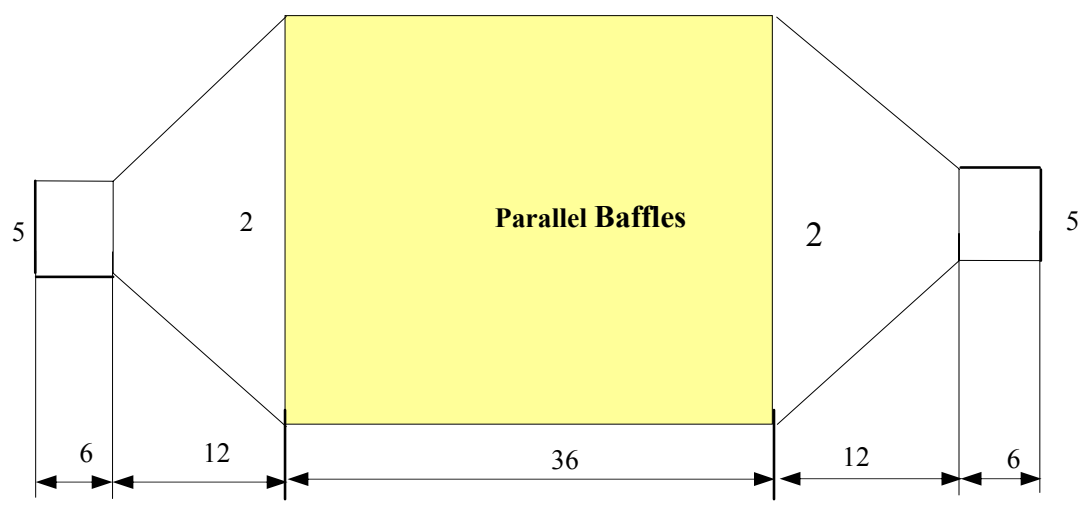

(a) exterior geometry (dimensions in inches)
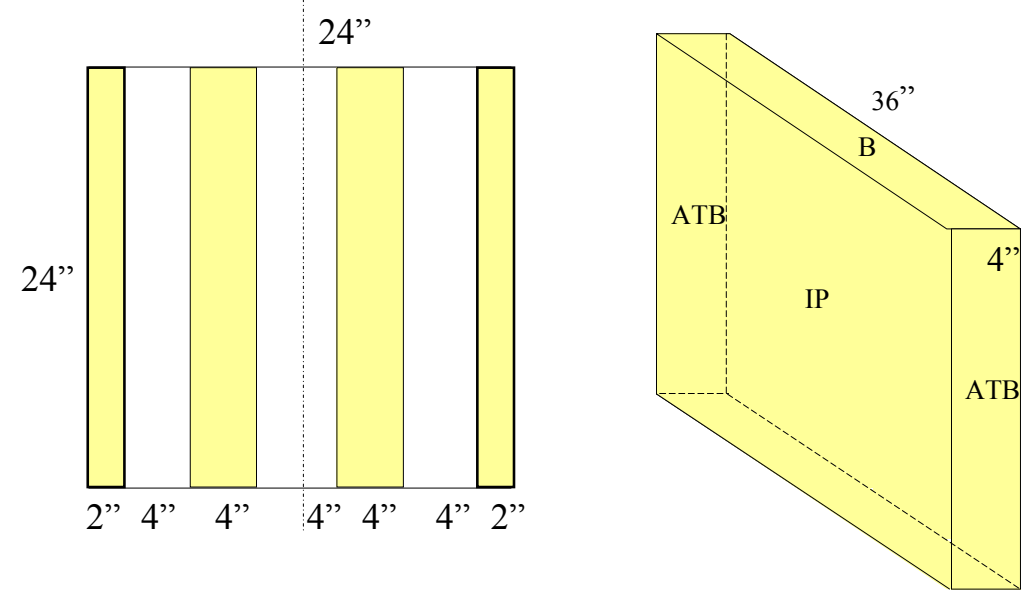

(b) cross section of the parallel baffles

(c) splitter geometry

Figure 4 Parallel-baffle silencer with a lining on both side walls.

Using the same test configuration, the catalytic monolith is replaced by a ceramic wallflow filter monolith (a DPF) in the third test case [19]. The DPF monolith has a cell density of 100 CPSI and is also uncoated. Figure 9 shows the BEM predictions versus the experimental data. It can be seen that both numerical approaches under-predict the TL values though the trend of the curves agrees well with the experimental results. The discrepancy is due to the fact that sound propagation in the wall-flow monolith is not 
limited to only the axial direction along the capillaries. There is significant amount of sound transmission through the walls of the filter monolith.

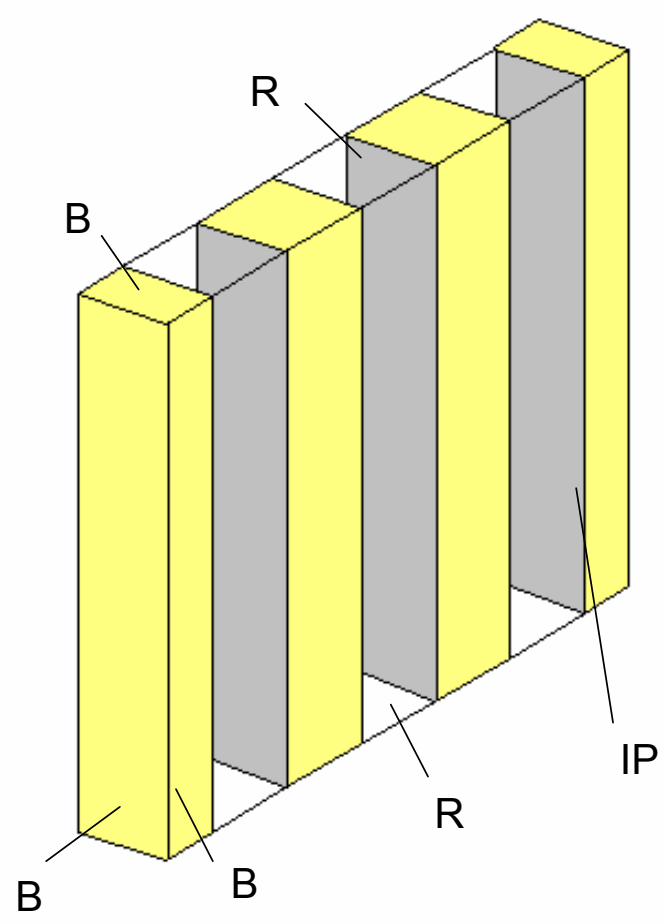

Figure 5 A substructure template created from the identical center portion of the parallel-baffle silencer in Fig. 4.

\section{Conclusions}

Recent developments of the direct mixed-body BEM for muffler and silencer analysis are reviewed. A key ingredient in the direct mixed-body BEM is the hypersingular integral equation. It is well known that the hypersingular integral equation is ideally suited to thin-body problems so that a multi-domain approach can be avoided. The direct mixed-body BEM has demonstrated that even a two-medium or multi-medium problem can benefit from the hypersingular integral equation.

Ironically, the direct mixed-body BEM, originally meant to the "terminator" of the old multi-domain BEM, has turned out to be a "facilitator" of the new substructuring technique which is basically a modular implementation of the old multi-domain BEM. For large structures, the combined technique of the direct mixed-body BEM and substructuring has proven to be very effective. 
This paper also presents two examples of mufflers with an emission control device such as a CC or DPF. Although the proposed four-pole matrix is very effective for the $\mathrm{CC}$ test case, it only provides an approximation for the DPF test case. More research is in order for the modeling of a DPF in the future.

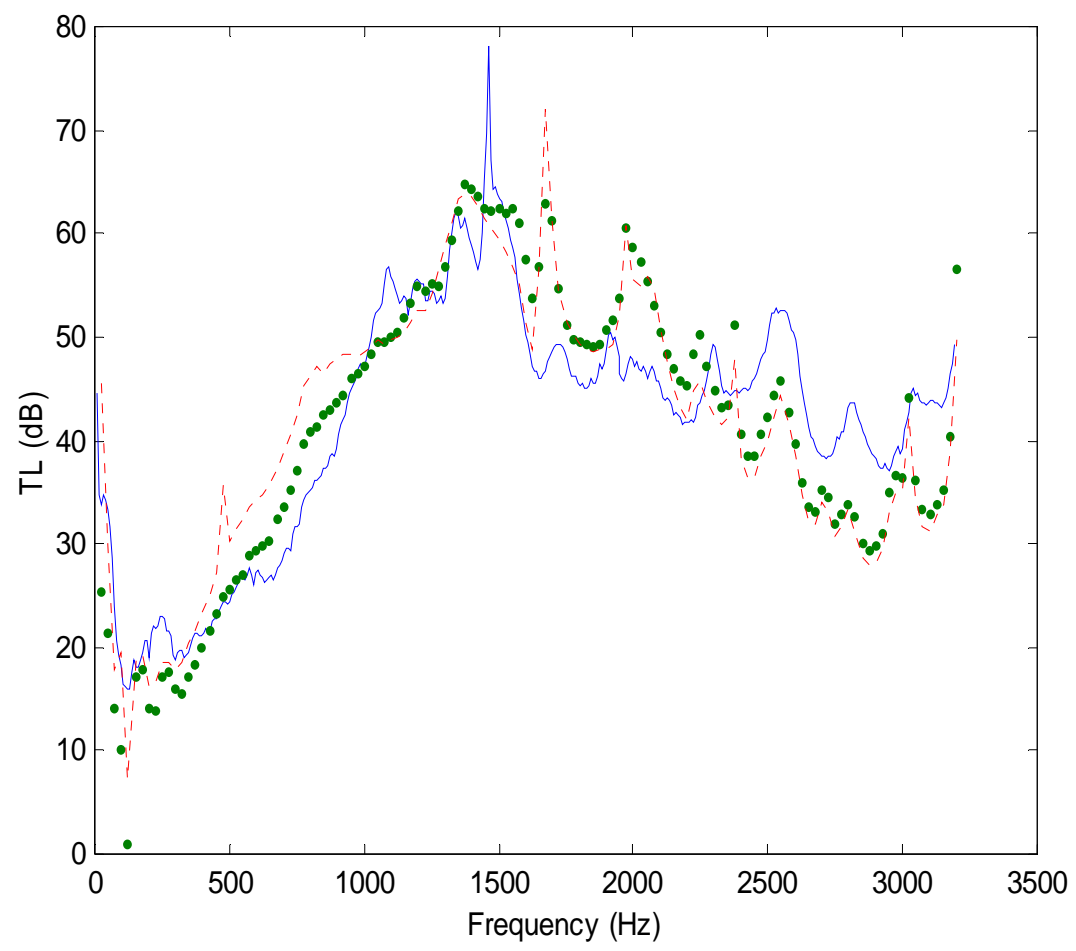

Figure 6 Transmission loss for the parallel-baffle silencer. Solid line: experiment; solid dots: BEM using four substructures; dotted line: BEM using ten substructures. 


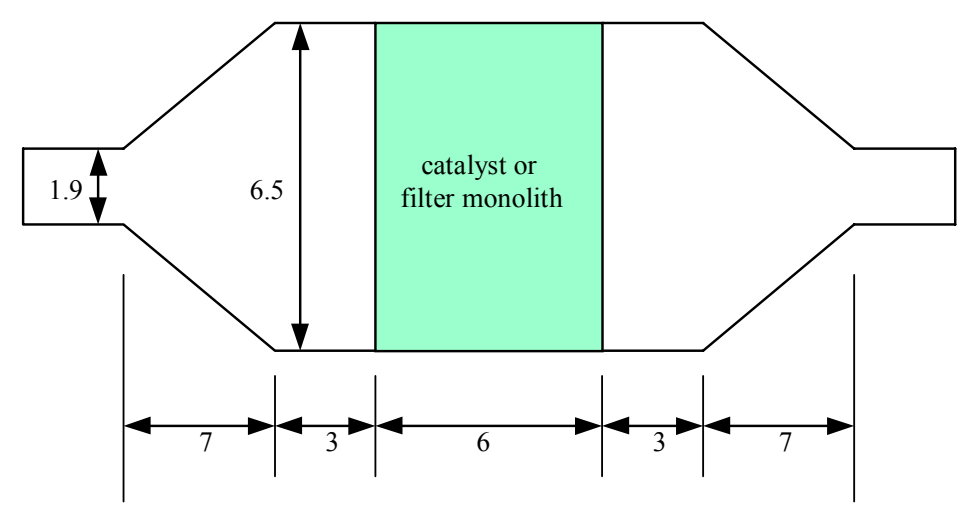

Figure 7 Emission control device test case (dimensions in inches).

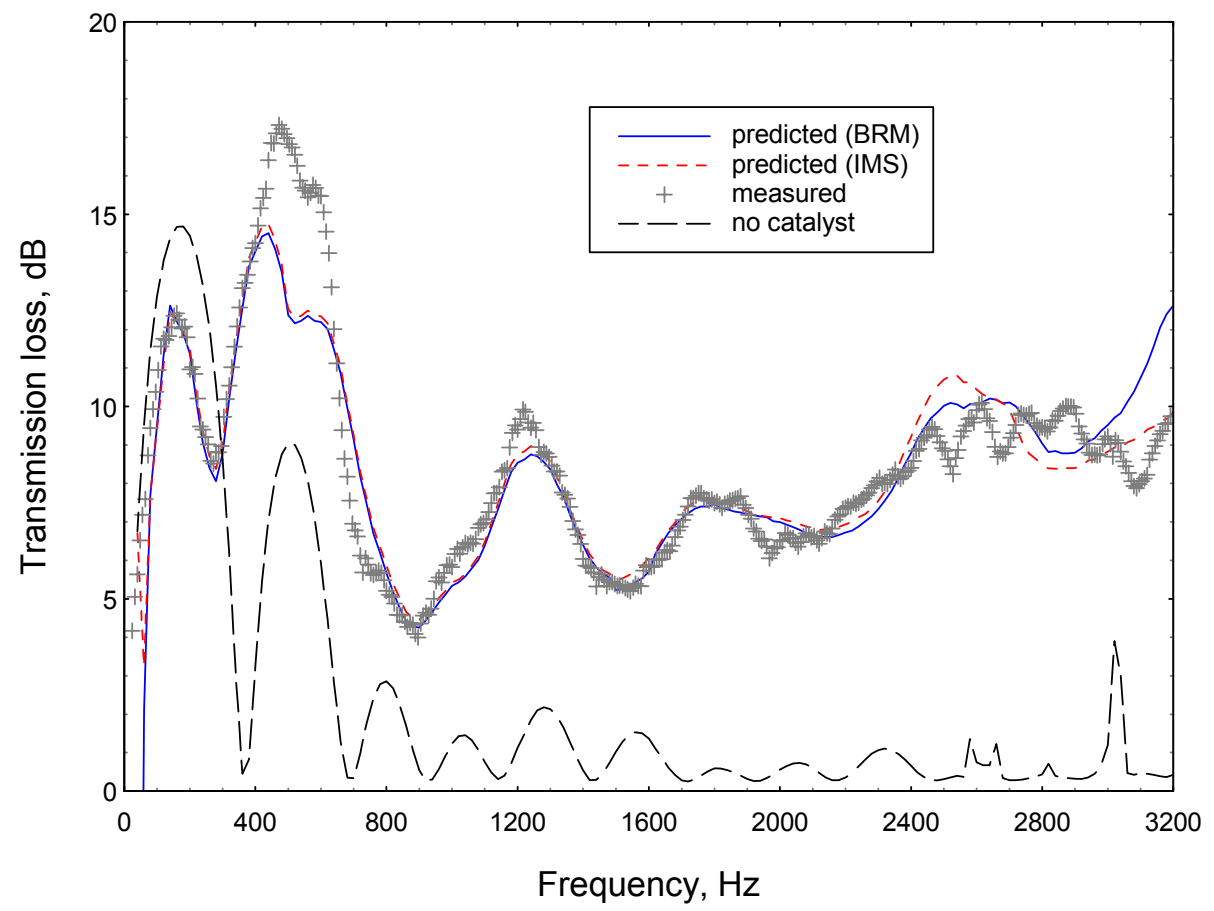

Figure 8 Comparison of the predicted and measured TL of the CC test case. 


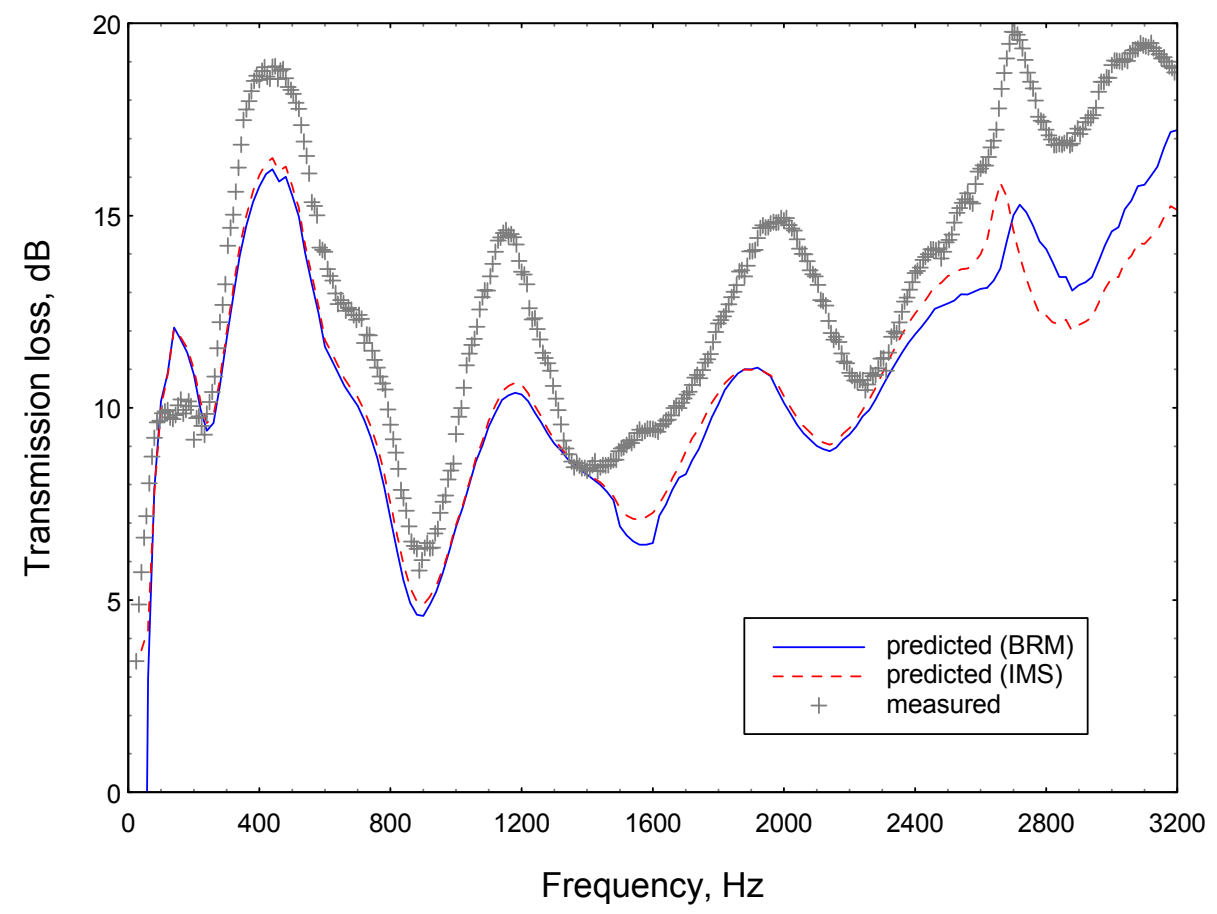

Figure 9 Comparison of the predicted and measured TL of the DPF test case.

\section{Acknowledgement}

This research was supported by Nelson Industries (a division of Cummins, Inc.)

\section{References}

1. Wu, T. W., Wan, G. C. Muffler performance studies using a direct mixed-body boundary element method and a three-point method for evaluating transmission loss. Journal of Vibration and Acoustics, ASME Transactions 1996; 118:479-484.

2. Wu, T. W., Zhang, P., Cheng, C. Y. R. Boundary element analysis of mufflers with an improved method for deriving the four-pole parameters. Journal of Sound and Vibration 1998; 217: 767-779.

3. Terai, T. On calculation of sound fields around three dimensional objects by integral equation methods. Journal of Sound and Vibration 1980; 69: 71-100. 
4. Krishnasamy, G., Schmerr, L. W., Rudolphi, T. J., and Rizzo, F. J. Hypersingular boundary integral equations: some applications in acoustic and elastic wave scattering. Journal of Applied Mechanics, ASME Transactions 1990; 57: 404-414.

5. Liu, Y. J. and Rizzo, F. J. A weakly singular form of the hypersingular boundary integral equation applied to 3-D acoustic wave problems. Computer Methods in Applied Mechanics and Engineering 1992; 96: 271-287.

6. Guiggiani, M., Krishnasamy, G., Rudolphi, T. J., and Rizzo, F. J. A general algorithm for the numerical solution of hypersingular boundary integral equations. Journal of Applied Mechanics, ASME Transactions 1992; 59: 604-614.

7. Liu, Y. J. and Rizzo, F. J. Hypersingular boundary integral equations for radiation and scattering of elastic waves in three dimensions. Computer Methods in Applied Mechanics and Engineering 1993; 107: 131-144.

8. Krishnasamy, G., Rizzo, F. J., and Liu, Y. J. Boundary integral equations for thin bodies. International Journal for Numerical Methods in Engineering 1994; 37: 107-121.

9. Chao J. C., Liu Y. J., Rizzo F. J., Martin, P. A., and Udpa, L. Regularized integral equations and curvilinear boundary elements for electromagnetic wave scattering in three dimensions. IEEE Transaction on Antennas and Propagation 1995; 43:1416-1422.

10. Martin, P. A. and Rizzo, F. J. Hypersingular integrals: How smooth must the density be? International Journal for Numerical Methods in Engineering 1996; 39: 687-704.

11. Sullivan, J. W., Crocker, M. J. Analysis of concentric-tube resonators having unpartitioned cavities. Journal of Acoustical Society of America 1978; 64:207-215.

12. Utsuno, H., Tanaka, T., Fujikawa, T., Seybert, A. F. Transfer function method for measuring characteristic impedance and propagation constant of porous materials. Journal of Acoustical Society of America 1989; 86:637-643.

13. Beranek, L. L., Editor, Noise and Vibration Control, Institute of Noise Control Engineering, 1988.

14. Utsuno, H., Wu, T. W., Seybert, A. F., Tanaka, T. Prediction of sound fields in cavities with sound absorbing materials. AIAA Journal 1990; 28:1870-1876.

15. Wu, T. W., Cheng, C. Y. R., Zhang, P. A direct mixed-body boundary element method for packed silencers. Journal of Acoustical Society of America 2002; 111: 2566-2572. 
16. Wu, T. W., Cheng, C. Y. R., Tao Z. Boundary element analysis of packed silencers with protective cloth and embedded thin surfaces. Journal of Sound and Vibration 2003; 261:1-15.

17. Ji, Z., Ma, Q., Zhang, Z. Application of the boundary element method to predicting acoustic performance of expansion chamber mufflers with mean flow. Journal of Sound and Vibration 1994; 173:57-71.

18. Lou, G., Wu, T. W., and Cheng, C. Y. R. Boundary element analysis of packed silencers with a substructuring technique. Engineering Analysis with Boundary Elements 2003; 27: 643-654.

19. Cheng, C. Y. R., Wu, T. W. and Lou, G. Acoustical performance prediction of exhaust emission control devices using BEM. Inter-Noise 2002, 2002; Paper $\mathrm{N} 125$, on CD. 